\title{
A Clinicopathological Study on Cyclical Mastalgia with Special Reference to Serum Oestrogen Level and Breast Volume
}

\author{
Amit Roy ${ }^{1}$, Prasenjit Chattopadhyay², Satya Prakash Kuila ${ }^{3}$, Ankit Agarwal ${ }^{4}$ \\ ${ }^{1}$ Department of General Surgery, Midnapore MC\&H, Paschim, Medinipur, West Bengal, India. \\ ${ }^{2}$ Department of Plastic Surgery, R. G. Kar Medical College and Hospital, Kolkata, West Bengal, India. \\ ${ }^{3}$ Department of General Surgery, Midnapore MC\&H, Paschim, Medinipur, West Bengal, India. \\ ${ }^{4}$ Department of General Surgery, Midnapore MC\&H, Paschim, Medinipur, West Bengal, India.
}

\section{ABSTRACT}

\section{BACKGROUND}

Cyclic mastalgia is defined as a breast pain that has a relationship to the menstrual cycle. As far as research studies are concerned the main etiological factors for cyclical mastalgia include dietary factors and hormonal imbalance. In this study, the clinicopathological aspect of cyclical mastalgia is studied with special reference to serum oestrogen and breast volume. This study provides valuable data on cyclical mastalgia with special reference to serum oestrogen level and breast volume.

\section{METHODS}

A total of 101 patients meeting inclusion criteria were selected for this prospective comparative observational study. Sample was selected from OPD patients from Surgery Department, Medical College Kolkata. A detailed history of the patients who was taken with special reference to the dietary habits and lifestyle. A thorough physical examination including breast size and volume were taken (by visual assessment method, size of the undergarments the patient wear and then breast circumference measurement) and the findings were noted. Patients underwent TruCut biopsy for histopathological study and Oestrogen Receptor Status. All data was captured in a predesigned Case Data Sheet and analysis of all the data was done from the CDS using master chart or grand chart.

\section{RESULTS}

$58.3 \%$ patients with cyclical mastalgia have h/o fatty diet. Patients with increased breast volumes had increased levels of serum oestradiol which signifies that mastalgia with medium and large size breast would respond to antioestrogens better than those patients with small sized breast volume. Majority of the patients with cyclical mastalgia in our study had medium breast volumes as per Bra-size, where the range of 32-36 has the highest frequency (42.8\%). The mean measurement of bra size is 33.7 and the standard deviation is 3.737. In our study, we find positive correlation with breast volume and serum oestrogen level (Pearson correlation of breast volume as 1 and serum oestrogen level as 0.381 respectively) and negative correlation with breast volume and serum progesterone level (Pearson correlation of breast volume as 1 and serum progesterone level as -0.134 respectively).

\section{CONCLUSIONS}

This study shows a significant positive correlation between breast volume, fatty diet and oestrogen level with cyclical mastalgia.

\section{KEY WORDS}

Cyclical Mastalgia, Serum Oestrogen, Breast Volume
Corresponding Author: Dr. Ankit Agarwal, Postgraduate Trainee, Midnapore Medical College, Paschim, Medinipur, West Bengal, India.

E-mail: ankit_aagarwal@hotmail.com

DOI: $10.14260 /$ jemds/2020/387

Financial or Other Competing Interests: None.

How to Cite This Article:

Roy A, Chattopadhyay P, Kuila SP, et al. A clinicopathological study on cyclical mastalgia with special reference to serum oestrogen level and breast volume. J. Evolution Med. Dent. Sci. 2020;9(23):1765-1770, 10.14260/jemds/2020/387 DOI:

Submission 12-01-2020,

Peer Review 12-05-2020,

Acceptance 19-05-2020,

Published 08-06-2020.

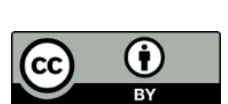




\section{BACKGROUND}

The breast or mammary gland is a distinguishing feature of the class Mammalia. The mature breast of the female extends from the level of second rib to the infra-mammary fold at the level of sixth or seventh rib. When fully developed, the female adult breast is composed of 15-20 lobes of branching gland. There is lots of fat tissue within the breast. The amount of fat determines the size of the breast. Cyclical mastalgia is very common in women and it interfere well between the daily routine activities, and still the confirmed etiological factors and primary treatment remained undefined. Breast pain is classified into cyclic mastalgia, noncyclic mastalgia, and extramammary (non-breast) pain according to their nature.[1] Cyclic mastalgia is breast pain that has a clear relationship to the menstrual cycle. Noncyclic mastalgia is a constant or intermittent pain and show no or less relationship with menstrual cycle and often occurs after menopause. Patient present with only symptom of pain have a very low chance of cancer and associated factors, symptoms and sign must be excluded well before making a primary diagnosis of cancer. A thorough examination should be done along with reassurance and pharmacological treatment and this provide basic treatment in maximum number of cases.

The medications like Danazol, tamoxifen, and Bromocriptine are effective; however, their potentially serious adverse effects limit their use to selected patients with severe, sustained breast pain.[2] Cyclical mastalgia, is associated with the menstrual cycle and is mainly hormonal. Many women have their pain started in the mid of the cycle that is during ovulation and continue till the end of the cycle till they menstruate. Type of pain may vary from women to women and it may be graded from very severe to mild. Severity may be such that in some cases they don't even allow for physical examination and tenderness present like breast abscess. Again, pain may vary according to quadrant as maximum women experience their pain in both breast which usually radiate to arm and axilla. To classify that pain is cyclical or not we have to precisely look for their relationship with menstrual cycle and nature of pain as cyclical mastalgia may be truly said after few months properly observing the menstrual cycle. Ongoing research process is there to establish the relationship between the cyclical mastalgia and hormonal level.[3] Few studies correlate cyclical mastalgia with oestrogen and progesterone hormone and their ratio while other studies correlate them with prolactin hormone. Other than hormonal level stress is also a contributing factor in cyclical mastalgia and moreover hormonal level itself change according to stress level, so they are better correlated.[4]

As far as research studies are concerned the main etiological factors for cyclical mastalgia include-

Dietary Factors- Tea/coffee and saturated fat have been suggested to be the cause of mastalgia. ${ }^{[4]}$

Hormonal Imbalance- Oestrogen excess, progesterone deficiency, changes in progestin/oestrogen ratio, differences in receptor sensitivity.

Disparate Secretion of Follicle- Stimulating hormone (FSH) and luteinising hormone (LH), low androgen levels, and high prolactin levels have all been implicated as the cause for mastalgia. However, evidence to confirm the causal relationship between mastalgia and various hormonal imbalance is lacking. In many studies it has been concluded that hormonal change is the main culprit for cyclical mastalgia but contradictory to this breast pain is equal in both breast and sometimes its unilateral and limited to one breast only. Thus, hormonal imbalance may not be concluded the only cause of cyclical mastalgia, and it seems that breast act differently to hormone level and thus a detail study is needed to conclude the exact etiological factor for cyclical mastalgia. A detail research work is needed in this order to draw a proper conclusion. Thus, in the present study A observational study is being carried out to detect the aetiology of cyclical mastalgia with special reference to serum oestrogen and progesterone level and breast volume.

\section{Significance of Breast Volume Measurement}

- As suggested by name the first use of breast volume measurement is to detect the size of breast and rule out macromastia. ${ }^{[5]}$

- To establish the indication for cosmetic surgery and prophylactic surgery in case of macromastia and to evaluate the size of specimen.

- Breast volume measurement provide a preoperative basis for breast conserving surgeries. ${ }^{[6],[7]}$

Breast volume measurement is a determining factor for selecting the approach to be used in all types of breast surgery (reduction, augmentation, reconstructive, and oncoplastic) in order to obtain symmetry of both breasts.[5],[8]

\section{METHODS}

The study began after receiving approval from Institutional Ethics Committee of Medical College, Kolkata. A predesigned Informed Consent Form (ICF) was given to every patient under the study and a valid, legal consent was taken from each of them. The study sample was selected on basis of OPD patients from Surgery Department, Medical College Kolkata. A detailed history of the patients who were in the criteria was taken with special reference to the dietary habits and other lifestyles. A thorough physical examination including breast size and volume were taken (by visual assessment method, size of the undergarments the patient wear and then breast circumference measurement) and the findings will be noted. The patient was explained in detail about the disease and blood biochemical parameters were assessed.

The patients had undergone Tru-Cut biopsy for histopathological and oestrogen Receptor Status. All data was captured in a predesigned Case Data Sheet and analysis of all the data was done from the CDS using master chart or grand chart. A study period of 18 month is taken into consideration that is from January 2014 to June 2015. It was an institutional based observational study conducted in Department of General Surgery, Medical College \& Hospital, Kolkata. 


\section{Study Parameters}

Different study tools ranging from history taking to Corecut Biopsy- all of which made and important contribution to the study need a brief but special mention.

\section{History Taking}

A careful, thorough and detailed history was taken regarding the socio- demographic profile - name, age, religion, occupation, residence, socio- economic scale (based on modified B.G. Prasad's scale), regarding past and family history - history of similar episodes in the past, history of similar episodes in the family, history of breast cancer in the family, regarding menstrual and obstetrics history- age of menarche, age of first child birth, number of child births, history of heaviness of breast during menstruation, history of taking OCP, history of hysterectomy, and regarding diet history - intake of high tea and coffee, history of high fatty food.

\section{Clinical Examination}

It includes various aspects of general survey and brief assessment of allied systemic examination. In local examination of breast, every detail of the breast was noted. In inspection, any skin or colour change and redness and inflammation was noted. In palpation, the findings of the inspection were confirmed. Apart from that tenderness, quadrant of maximum tenderness was noted.

\section{Measurements}

Some measurements were very much important in our study. Few of them were height in centimetre and weight in kilograms which were required to measure the Body Mass Index. Moreover, breast circumference measurements taking proper permission from the patient was done by non-elastic plastic measuring tape. This was required to evaluate the breast circumference of the mastalgia patients.[9][10]

\section{Radiological Examination}

Mammographic examination was done to note the BIRADS.

\section{Biochemical Examination}

Blood tests for serum oestradiol, serum progesterone, and lipid profile including Serum cholesterol, triglycerides and low-density lipoprotein were done to evaluate their values.

\section{Pathological Examination}

TruCut Biopsy was done in all the patients who came out with the histopathology report and the oestrogen Receptor Status during follow ups.

\section{Sample Size}

A total of 101 patients with complain of cyclical mastalgia.
Prevalence of Cyclical mastalgia $(\mathrm{P})=3 \%(0.03)$ in the Indian population. Considering the prevalence, the minimum number of samples required for the study comes out to be 81 , applying the below mentioned formula-

$$
\frac{(\mathrm{Z} \alpha)^{2} \mathrm{pq}}{\mathrm{L}^{2}}
$$

where $\mathrm{p}=0.03$

$\mathrm{q}=1-\mathrm{p}=0.07$

$\mathrm{L}=10 \%$ absolute error

$\mathrm{Z}_{\alpha}=1.96[95 \%$ Confidence Limit $]$

Since patients were needed to be followed up for collection of biochemical and pathological data, there were chances of dropouts / attritions.

Estimating 25\% attrition rate, 101 patients needed to be sampled. Hence my sample size was 101 .

\section{Inclusion Criteria}

- Pre-menopausal and at least 18 years of age.

- Has a history of cyclical mastalgia for each of the four months prior to study entry.

- Has no history of non-cyclical and extramammary mastalgia.

- Has a history of regular menstrual cycles of $28 \pm 3$ days.

\section{Exclusion Criteria}

- Having cyclical mastalgia with prominent breast lumps (like fibroadenoma).

- Having breast implants.

- Having suspected breast disease other than mastalgia (like breast malignancy, breast abscess, antibioma, etc.)

- Having any other co morbidities.

- Having any analgesic medications prior to the study.

- Nursing mother within 3 months of study.

- Pregnant.

\section{Study Design}

A prospective observational study.

\section{Study Tools}

Non-elastic plastic tape, Paper, Pen, Liquid Hand wash, Sterile gloves, Disposable gloves, Calculator, Computer.

\section{Statistical Analysis}

A total of 101 patients with cyclical mastalgia were taken into study. All the patient underwent detailed history and clinical evaluation, biochemical, radiological and histopathological examination. Out of 101 patients sampled, only 96 of them were turned up in the Surgery OPD with full data and reports on follow up visits. Final results were based on 96 patients with cyclical mastalgia. Data were entered and analysed with the help of MS Excel EPI info (TM) 7.2.2.2. EPI INFO is a trademark of the centers of disease control and prevention 
(CDC). To find out the correlation between breast volume, serum oestradiol level and serum progesterone level, Pearson Correlation coefficient is used. Quantitative or continuous variables were presented as mean \pm SD and compared using student ' $\mathrm{t}$ ' test. $\mathrm{p}<0.05$ was considered as statistically significant.

\section{RESULTS}

Patients in my study have a negative correlation of heaviness of breast during menstruation and cop intake. The mean value of breast volume is 377.722 cc with standard deviation of 189.218 when compared in 96 patients. The mean measurement of bra size is 33.7 and the standard deviation being 3.737 . It is observed in our study that the Breast volume has a positive correlation with serum oestradiol level and a negative correlation with serum progesterone level. Also, it is observed that both serum oestradiol and serum progesterone level have negative correlations among each other. In the present prospective study, we have focused on serum E2 concentration in benign breast disease and found them to be significantly higher in Fibroadenosis than in FCC patients $(\mathrm{p}<0.001)$. We found that E2 levels varied among different histopathological variants being higher in fibroadenosis and lesser in fibrocystic disease patients. Moreover, we found that the means E2 levels higher in patients with positive ER status than the patients with negative ER status.

\begin{tabular}{|c|c|c|}
\hline & No. of Patients & Percentage \\
\hline \multicolumn{3}{|c|}{ Past h/o Prolonged Intake of OCP } \\
\hline$+v e$ & 20 & 20.8 \\
\hline -ve & 76 & 79.2 \\
\hline Total & 96 & 100 \\
\hline \multicolumn{3}{|c|}{ Past h/o Hysterectomy } \\
\hline$+\mathrm{ve}$ & 5 & 5.2 \\
\hline -ve & 91 & 94.8 \\
\hline Total & 96 & 100 \\
\hline \multicolumn{3}{|c|}{ h/o Heaviness of Breast during Menstruation } \\
\hline$+v e$ & 60 & 62.5 \\
\hline -ve & 36 & 37.5 \\
\hline Total & 96 & 100 \\
\hline \multicolumn{3}{|c|}{$\begin{array}{l}\text { Table 1. Distribution in Relation to } \\
\text { Obstetric and Menstrual History }\end{array}$} \\
\hline
\end{tabular}

\begin{tabular}{|cccc|}
\hline & Mean & Standard Deviation & n \\
Breast Volume (cc) & 377.722 & $\mathbf{1 8 9 . 2 1 8}$ & 96 \\
& & \\
\hline \multicolumn{3}{|c|}{ Table } & 2. Breast Volume Measurement \\
\hline
\end{tabular}

\begin{tabular}{|c|c|c|c|c|c|}
\hline$N=96$ & & $\begin{array}{c}\text { Breast } \\
\text { Volume }\end{array}$ & $\begin{array}{c}\text { Serum } \\
\text { Estradiol }\end{array}$ & $\begin{array}{c}\text { Serum } \\
\text { Progesterone }\end{array}$ & Mean \pm SD \\
\hline $\begin{array}{c}\text { Breast } \\
\text { Volume }\end{array}$ & $\begin{array}{l}\text { Pearson } \\
\text { Correlation }\end{array}$ & 1 & 0.381 & -0.134 & $377.72 \pm 189.22$ \\
\hline $\begin{array}{c}\text { Serum } \\
\text { Oestradiol }\end{array}$ & $\begin{array}{l}\text { Pearson } \\
\text { Correlation }\end{array}$ & 0.381 & 1 & -0.330 & $257.06 \pm 234.62$ \\
\hline $\begin{array}{c}\text { Serum } \\
\text { Progesterone }\end{array}$ & $\begin{array}{l}\text { Pearson } \\
\text { Correlation }\end{array}$ & 0.191 & 0.001 & 1 & $21.71 \pm 33.53$ \\
\hline
\end{tabular}

In this study, majority of the patients gave positive history of heaviness of breast during menstruation (60) and negative history of taking prolong OCPs (76) and negative history of hysterectomy in the past (91). It is observed in our study that the Breast volume has a positive correlation with serum oestradiol level and a negative correlation with serum progesterone level. Also, it is observed that both serum oestradiol and serum progesterone level have negative correlations among each other. In my study, we have found that ER +ve patients were more in percentage in fibroadenosis group of patients than patients with fibrocystic change. In the present prospective study, we have focused on serum E2 concentration in benign breast disease and found them to be significantly higher in Fibroadenosis than in FCC patients $(\mathrm{p}<0.001)$. We found that E2 levels varied among different histopathological variants being higher in fibroadenosis and lesser in fibrocystic disease patients. Moreover, we found that the means E2 levels higher in patients with positive ER status than the patients with negative ER status.

\begin{tabular}{|ccc|}
\hline ER Status & Types of BBD \\
ER $+\mathrm{ve}$ & $51(63 \%)$ & FCC $(\mathbf{n}=14)$ \\
ER -ve & $31(37 \%)$ & $3(21 \%)$ \\
\hline \multicolumn{2}{|c|}{ Table 4. Comparison of Oestrogen Receptor Status in } \\
Different Types of Benign Breast Diseases
\end{tabular}

\begin{tabular}{|cc|}
\hline ER Status & Serum Oestradiol Level (Mean Level) \\
ER +ve & $263 \pm 3.56$ \\
ER -ve & $133 \pm 5.89$ \\
& Histopathological Types \\
Fibroadenosis & $190 \pm 7.64$ \\
FCC & $110 \pm 8.12$ \\
\hline Table 5. Comparison of ER Receptor and Histopathological \\
Variants with Mean Serum Oestradiol Level
\end{tabular}

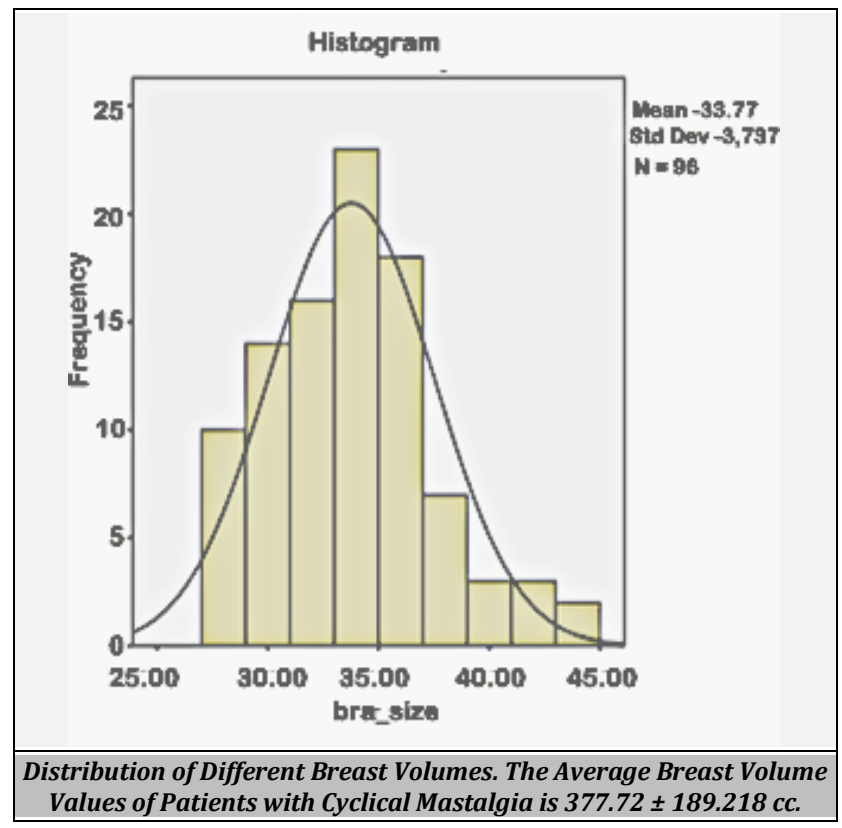

Majority of the patients with cyclical mastalgia in our study had medium breast volumes as per bra-size, where the range of 32-36 has the highest frequency (41). The mean measurement of bra size is 33.7 and the standard deviation being 3.737 .

\section{DISCUSSION}

Our study aims to review the assessment of various socio demographic, etiological, clinical, biochemical, radiological and histopathological factors with cyclical mastalgia. Also, to correlate the effect of different breast volumes and serum 
oestrogen levels and oestrogen receptor levels with cyclical mastalgia. In my study, (37) patients are detected to be ER positive status and rest (59) are detected to be ER negative status out of total (96) study population. We have found that ER +ve patients were more in percentage in fibroadenosis group of patients than patients with fibrocystic change. Maximum subset of patients in my study area were found to be of Hindu religion (74\%) followed by Muslim (24\%) and lastly Christian (2\%). No other community were present in my study population. In this study, the study population from both, rural and urban regions are more or less equal with a slight preponderance to urban population in relation to the rural one. In my study married females (69.8\%) are more affected with cyclical mastalgia than unmarried females. Most patients with cyclical mastalgia fell into the age group of 25-34 years (36.46\%), followed by age group of 35-44 years $(34.37 \%)$ and the least with lower age group of $<25$ yrs. (29.17\%). In our study cyclical mastalgia is mostly prevalent in nulliparous women $(35.4 \%)$, followed by women having 2 children (31.2\%), 1 child $(27.1 \%)$, and 3 children $(6.3 \%)$ respectively. Here in this study it is seen that the majority of the patients with cyclical mastalgia had menarche age group of 11-13 yrs, followed by age group of less than 10 yrs. In this study, it is found that majority of the patients with cyclical mastalgia has positive history of high tea (50), coffee (49) and fatty food (56) intake as their food habit. In this study, only 3 patients had palpable axillary lymph nodes while others (93) were node free during examination. Majority of the patients with cyclical mastalgia in our study had medium breast volumes as per brasize, where the range of 32-36 has the highest frequency. The mean measurement of bra Size is 33.7 and the standard deviation being 3.737. In our Study, maximum patients (72) has BIRADS 1 on mammographic findings, followed by BIRADS 2 with patients and BIRADS 3 patients. There were no patients with BIRADS 0, BIRADS 4 or BIRADS 5. In our study, most patients with cyclical mastalgia (80) had diffuse tenderness of breast. Only 16 of them had localised tenderness of breast, out of which, upper outer quadrant had the maximum prevalence (8), followed by upper inner (5), lower outer (2), lower inner (1). There were no patients with maximum tenderness localised in central and axillary tail area. In this study it is evident that majority of the patients with cyclical mastalgia are due to Fibroadenosis of breast followed by FCC (Fibrocystic Changes) of the breast.

Preece et al. in 1976 found that most frequent age group complaining of mastalgia was 31-45 years closely followed by 41-45 years, and 36-40 [47]. In another study by Wisbey et al. Welsh national school of medicine at Cardiff (1983) it was seen that in patients of cyclical mastalgia mean age was $35.7( \pm 8.7)$, whereas in patients with non-cyclical mastalgia mean age was $(42.5 \pm 9.8)$ years [48]. In our study, the study population was grouped into 3 groups $-<25$ yrs., 25-35 yrs., >35 yrs. Here, most patients with cyclical mastalgia fell into the age group of 25-34 years (36.46\%), closely followed by age group of 35-44 years (34.37\%) and the least with lower age group of $<25 \mathrm{yrs}$. $(29.17 \%)$. Thus, the findings were nearly corroborative with the other major studies in the literature.

Jeffcoate et al..[5] a study in 1975 said that cyclical mastalgia was a disease of "frustrated unhappy nullipara" showing that the disease was mainly predominant in young females without any children.[11] In my study married females (69.8\%) are more affected with cyclical mastalgia than unmarried females
(30.2\%). Moreover, in this study cyclical mastalgia is mostly prevalent in nulliparous women (35.4\%), followed by women having 2 children (31.2\%), 1 child (27.1\%) and 3 children (6.3\%) respectively. Hence these findings corroborate with other studies done previously A recent study by Peter et al. [72] in 2003 investigated morphological structures by ultrasound of 335 women in Germany, 212 of whom had breast pain. The intensity of pain showed a significant positive correlation with the width of the milk ducts, suggesting an association between duct ectasia and mastalgia. In our radiological study, we have performed U.S.G. for both breasts and axilla and mammography as a radiological investigation. The findings of U.S.G. matched with the previous study with nearly all the patients with mastalgia having prominent and dilated terminal duct system. In mammography majority of the patients within the study had BIRADS scoring of 1 (74\%), followed by $2(14.6 \%)$ and $3(10.4 \%)$.

It is observed in our study that the Breast volume has a positive correlation with serum oestradiol level and a negative correlation with serum progesterone level. Also, it is observed that both serum oestradiol and serum progesterone level have negative correlations among each other. On histopathological examination, maximum patients with cyclical mastalgia were found to have fibroadenosis (85.4\%) with the rest having FCC (14.6\%). As patients with definite lumps were excluded, no fibroadenoma patients were taken into consideration. This study was found in concordance with study by Khanna et al.[12][13] where maximum is patient is of fibroadenoma, fibroadenosis and FCC. In her study immuno histochemical studies for ER in the tissue sample revealed the highest and most intense positivity among fibroadenoma patients as compared to fibroadenosis and fibrocystic disease. Among fibroadenoma patients 3+ ER status was seen in 22 (79\%), among fibroadenosis in 11 (50\%) and among fibrocystic disease in $3(30 \%)$ patients. On analysis, stronger ER positivity in tissues correlated with greater serum oestradiol levels $(\mathrm{p}<0.01)$. In the present prospective study, we have focused on serum E2 concentration in benign breast disease and found them to be significantly higher in fibroadenosis than in FCC patients $(p<0.001)$. We found that E2 levels varied among different histopathological variants being higher in fibroadenosis and lesser in fibrocystic disease patients. Moreover, we found that the means E2 levels higher in patients with positive ER status than the patients with negative ER status.

\section{CONCLUSIONS}

This study shows a positive significant correlation between breast volume, fatty diet and oestrogen level with cyclical mastalgia. After analysis we have concluded that patients with medium sized breasts having bra sizes of 32-36 has the highest frequency of cyclical mastalgia. The mean breast volume was found to be $377.72 \pm 189.218$ cc. It is observed in our study that the breast volume has a positive correlation with serum oestradiol level and a negative correlation with serum progesterone level. Also, it is observed that both serum oestradiol and serum progesterone level have negative correlations among each other. Hence from this observational study, we can conclude that with increased breast volumes, 
increased levels of serum oestradiol were noted which indicates that mastalgia with medium and large size breast volume would respond to antioestrogens better than those patients with small sized breast volume. In the study population, on comparing the histopathological variant, we found that the fibroadenosis population surpassed that of the fibrocystic change category. Moreover, majority of the fibroadenosis were in ER +ve domain and higher mean oestradiol level than the FCC population. Patients in fibroadenosis group of cyclical mastalgia will respond earlier and better with antioestrogens than the FCC variety. With 1 unit increase in breast volume, odds of ER status positivity increase significantly.

The present study was conducted with a small sample. Yet it may initiate the fulfilment of its mission if medical and anthropological investigators continue the work by establishing normative data of face. Data for different regions of India and Asia was lacking. For more accurate set of data, future studies should be performed on a larger sample.

\section{REFERENCES}

[1] Tezel E, Numanoglu A. Practical do-it yourself device for accurate volume measurement of breast. Plast Reconstr Surg 2000;105(3):1019-23.

[2] Kayar R, Catal H. Macromastia and breast cancer. Izmir Tepecik Hstn Derg 2007;17:121-30.

[3] Kovacs L, Eder M, Hollweck R, et al. Comparison between breast volume measurement using $3 \mathrm{D}$ surface imaging and classical techniques. Breast 2007;16(2):137-45.

[4] Tata JR. One hundred years of hormones. EMBO Reports 2005;6(6):490-6.

[5] Jeffcoate N, Principles of Gynaecology. $4^{\text {th }}$ edn. London: Butterworts 1975: p. 550.

[6] Brown RW, Cheng Y, Kurtay M. A formula for surgical modifications of the breast. Plast Reconstr Surg 2000;106(6):1342-5.

[7] Cochrane RA, Valasiadou P, Wilson ARM, et al. Cosmesis and satisfaction after breast-conserving surgery correlates with the percentage of breast volume excised. Br J Surg 2003;90(12):1505-9.

[8] Griffith CD, Dowle CS, Hinton CP, et al. The breast pain clinic: a rational approach to classification and treatment of breast pain. Postgrad Med J 1987;63(741):547-9.

[9] Kalbhen CL, McGill JJ, Fendley PM, et al. Mammographic determination of breast volume: comparing different methods. Am J Roentgenol 1999;173(6):1643-9.

[10] Grossman A, Roudner LA. A simple means for accurate breast volume determination. Plast Reconstr Surg 1980;66(6):851-2.

[11] Qiao Q, Zhon G, Ling Y. Breast volume measurement in young Chinese women and clinical applications. Aesthetic Plast Surg 1997;21(5):362-8.

[12] El-Oteify M, Megeed HA, Ahmed B, et al. Assessment of the breast volume by a new simple formula. Indian J Plast Surg 2006;39(1):13-1.

[13] Marshall J, Graham S, Swanson M. Caffeine consumption and benign breast disease: a case-control comparison. Am J Public Health 1982;72(6):610-2. 